\title{
ARTIGO
}

\section{A prevenção no atraso do desenvolvimento}

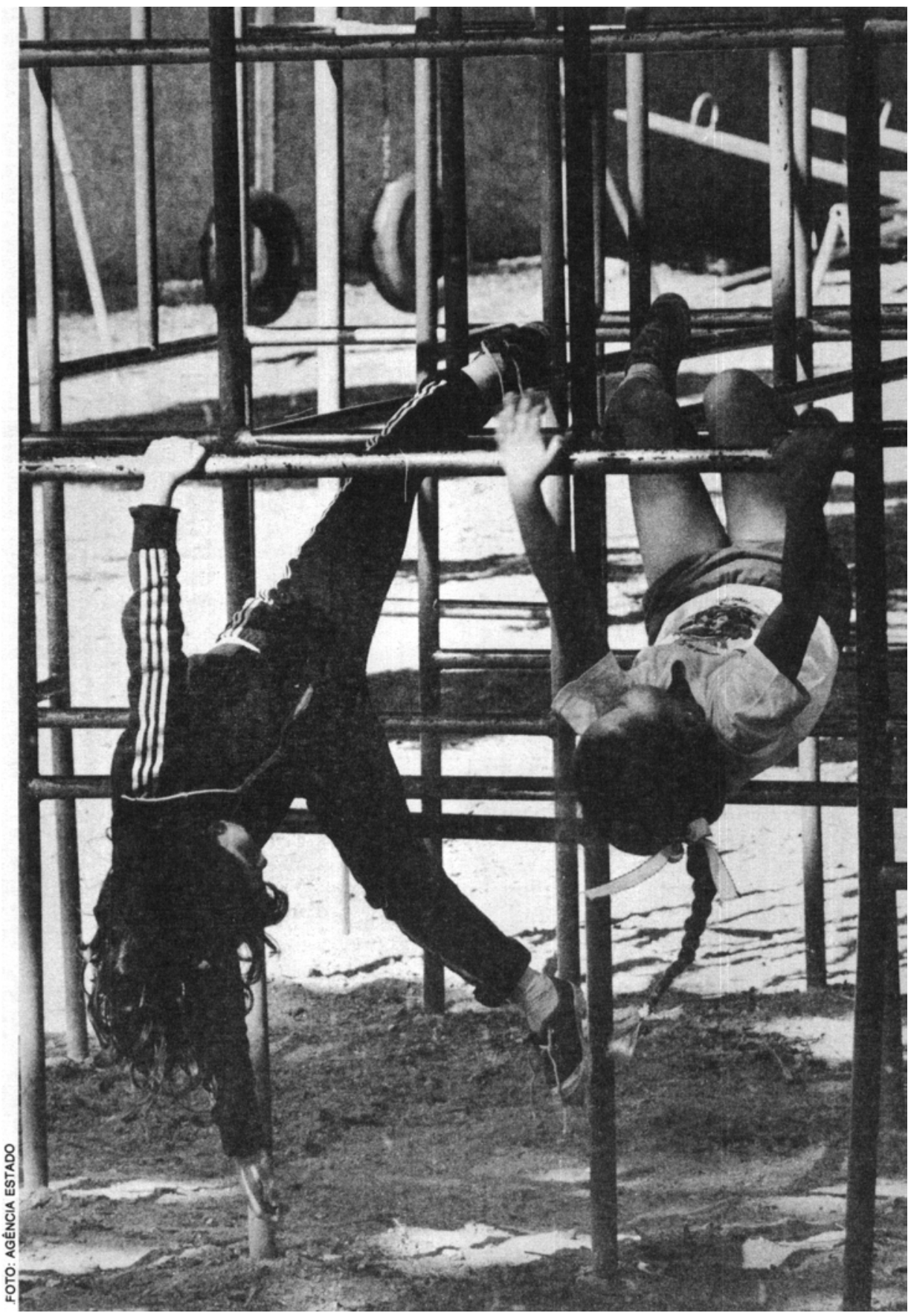

O presente trabalho teve como objetivo fazer uma revisão dos estudos realizados na área de intervençăo precoce.

É feita uma revisão dos diversos programas que visam a prevenção do atraso no desenvolvimento tendo sido eles subdivididos em três tipos, segundo o tipo de interverção adotada:

- numa creche, sem envolvimento da mãe

- numa creche ou em um centro e em casa, com envolvimento da mãe - em casa, com envolvimento da mãe

Finalmente, é apresentada uma análise das implicações desses programas de intervenção, em termos da abordagem adotada (intervenção sobre a criança $x$ intervenção sobre a interação mãe-filho), da utilização de testes de QI e do tipo de desenho experimental utilizado. Texto para "replacement"' sobre a questão.

Carolina Lampréio Pontificia Universidede Catolica do Rio de Janeiro

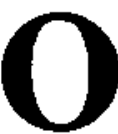
desenvolvimento da criança tem sido alvo de uma preocupa. ção crescente, baseada em teorias que consideram os primeiros anos de vida como fundamentais para o curso do desenvolvimento, o que tem levado à procura de um maior aprovejtamento de todo o potencial de aprendizagem da criança, antes de seu ingresso na escola. Isso tornou-se mais evidente quando, nos EUA, na década de 60, o governo decídiu adotar uma política social-educacional visando à "recuperação" das crianças de baixo 
status sócio-econômico, através do programa "Head Start".

A argumentaçāo subjacente é a de que o atraso no desenvolvimento das crianças em desvantagem deve ser atribuído, em grande parte, ao fato de terem crescido num ambiente carente de estímulos facilitadores do desenvolvimento. Tentar modificar esse atraso intervindo, pela primeira vez, ao nivel do ensino básico, é atuar muito tarde já que no primeiro ano primário espera-se que a criança tenha adquirido certo grau de discriminação auditiva e visual, certo nível de vocabulário e de coordenação motora, certo grau de desenvolvimento afetivo e social, que tenha exercitado sua capacidade de atenção e memória e que esteja motivada para a aprendizagem. Por isso; há a ênfase crescente para que se estimule e aperfeiçoe o processo de aprendizagem desde o nascimento. Parece fundamental para a futura vida escolar da criança, que ela ingresse no $1^{\circ}$ ano já possuindo as habilidades mínimas necessárias para que possa adquirir os novos comportamentos que dela serão exigidos. Sem isso será observada, o que não é incomum atualmente, uma deficiêencia cumulativa que acabará por não ser recuperada nunca mais. Esse parece ser o principal problema da educação em nossos dias. Como esperar que uma criança aprenda a escrever se ela ainda năo desenvolveu a coordenação motora necessária, que é um pré-requisito para que ela escreva? O problema não é que a criança não irá aprender a escrever por não possuir ainda a coordenaçăo motora necessária. $\mathrm{O}$ problema é que, enquanto ela ainda estiver desenvolvendo essa habilidade, sua turma estará seguindo adiante com novas aprendizagens e ela irá ficando para trás. A deficiência cumulativa decorre daí. É neste contexto que têm surgido os programas de intervenção precoce.

\section{PROGRAMAS DE INTERVENÇÂO PRECOCE}

A revisão dos programas de intervençâo precoce, que se segue, tomou como critério de classificação o local e o agente da intervenção sobre a criança: 1 - numa creche, sem envolvimento da mãe.

2 - numa creche ou em um centro e em casa, com envolvimento da mäe.

3 - em casa, com envolvimento da mãe.
1) Programas de intervençẫo realizados atraves de uma creche, sem envolvimento da mâe.

Foram realizados, na North Carolina Univestity, vários trabalhos de estimulação precoce, em creche, a partir de um programa básico de intervençāo, o "Carolina Abecedarian Project", cujo objetivo foi a "prevenção de um declínio no desenvolvimento intelectual de crianças de alto risco" (Ramey e Campbell, 1979-a). Neste programa, foram utilizado um grupo esperimental (GE constituído por sujeitos considerados de alto risco, a partir de um índice que incluiu nível de educação dos pais, rendimentos, QI materno, história de problemas sociais ou emocionais, evidência de retardo educacinal em outros membros da família, presença do pai em casa e necessidade de assistência pública) e dois grupos de controle (GC - sendo um constituído por sujeitos também de alto risco e o outro por sujeitos da população em geral). A pessoa treinada, neste caso, foi a própria criança $c o$ local de treinamento, a creche. $O$ procedimento de intervençào consistiu em atividades individuais, na sala de aula, que eram revistas a cada duas semanas, baseadas em dois currículos ("Carolina Infant Curriculum" e "Task Orientation Curriculum'), incluindo as áreas de linguagem, motora, social e cognitiva. A avaliação periódica dos sujeitos incluiu o

Bayley, um teste estandarizado de inteligência, o Stanford-Binet e a "McCarthy Scale of Intelligence".

Seguindo esse mesmo modelo de seleção, desenho experimental e intervençăo, Zeskind e Ramey (1978) procuraram "examinar a utilidade preditiva das características antropométricas, ao nascimento, para o desenvolvimento intelectual do bebê, em ambientes de cuidado pós-natais favoráveis e não-favoráveis". Para isto utilizaram, como instrumentos de avaliaçăo, o'Bayley, o Stanford-Binet, a "Maternal Involvement Subscale" do "Home Observation for Measurement of the Environment", os quais foram aplicados em 2 GE (bem nutrido $x$ mal nutrito fetalmente) e em 2 GC (com as mesmas características dos GE). Ramey e Campbell (1979-b) procuraram fazer "uma análise comparativa de grupos experimental e de controle, através do McCartny Scales of Children's Abilities que proporciona índices de funcionamento verbal, de desempenho perceptual, quantitativo, de memória e motor"; e Ramey, Farran e Campbell (1979) procuraram "avaliar as relaçōes entre características maternas e o desenvolvimento intelectual da criança, numa amostra homogênea de baixa ren- da, e determinar se essas relaçōes são alteradas por uma intervenção educacional precoce", através de avaliaçōes da mãe utlizando o WAIS ou WISC, a observaçāo de laboratório do comportamento mãe-fitho, o "Home Observation for Measurement of the Enviroment" $e$ o "Parental Attitudes Research Inventory".

Em sintese, os resultados mais relevantes desses trabalhos mostram que: - crianças, em desvantagem, submetidas a um programa de intervençāo, numa creche, sem o envolvimento da mãe, podem passar a apresentar um desenvolvimento dentro dos limites normais (Ramey e Campbell, 1979-a; Ramey e Campbell, 1979-b).

- as diferenças de desempenho entre os grupos experimental e de controle começam a surgir a partir dos 18 meses de idade (Ramey e Campbell, 1979-a; Zeskind e Ramey, 1978).

- as crianças em desvantagem começam a mostrar déficits, nos testes, na idade em que a linguagem falada começa a se desenvolver (Ramey e Campbell, 1979-a).

- bebês pouco responsivos pođem ter mães menos responsivas e sensiveis às suas açōes (Zeskind e Ramey, 1978).

- as características da mäe de uma criança em desvantagem têm uma influência menor, no desenvolvimento da criança, quando esta foi submetida a um programa de intervenção precoce (Ramey, Farran e Campbell, 1979).

2) Programas de intervenção realizados em um centro ou em um centro e em casa, com envolvimento da mãe

Os programas incluídos nesta categoria se diferenciam dos anteriores por utilizarem também as māes como agentes de intervenção para prevenir o atraso no desenvolvimento de seus filhos.

Em Ramey, Sparling, Wasik e Bryant (1979) procurou-se "comparar programas em centros e em casa, e examinar o processo de mudança que pode ser esperado tanto nas mães como nas crianças envolvidas nos programas", enquanto que em Hayden (1976); Frederick, Baldwin e Grove (1976); Wiegenrink e Parrish (1976); Garber (1977); Honig (1977); Jones (1977) e Gray e Klaus (1965) procurou-se intervir sobre a própria mãe, além do programa proporcionado pelo centro, onde as crianças compareciam quase que diariamente (de 4 a 5 dias/semana), durante todo o ano.

As características familiares e da criança e o critério de inclusăo no programa nem sempre estão bem definidos, mas pode-se dizer que, de uma maneira geral, procuraram atingir crianças de baixo nível sócio-econômico. 


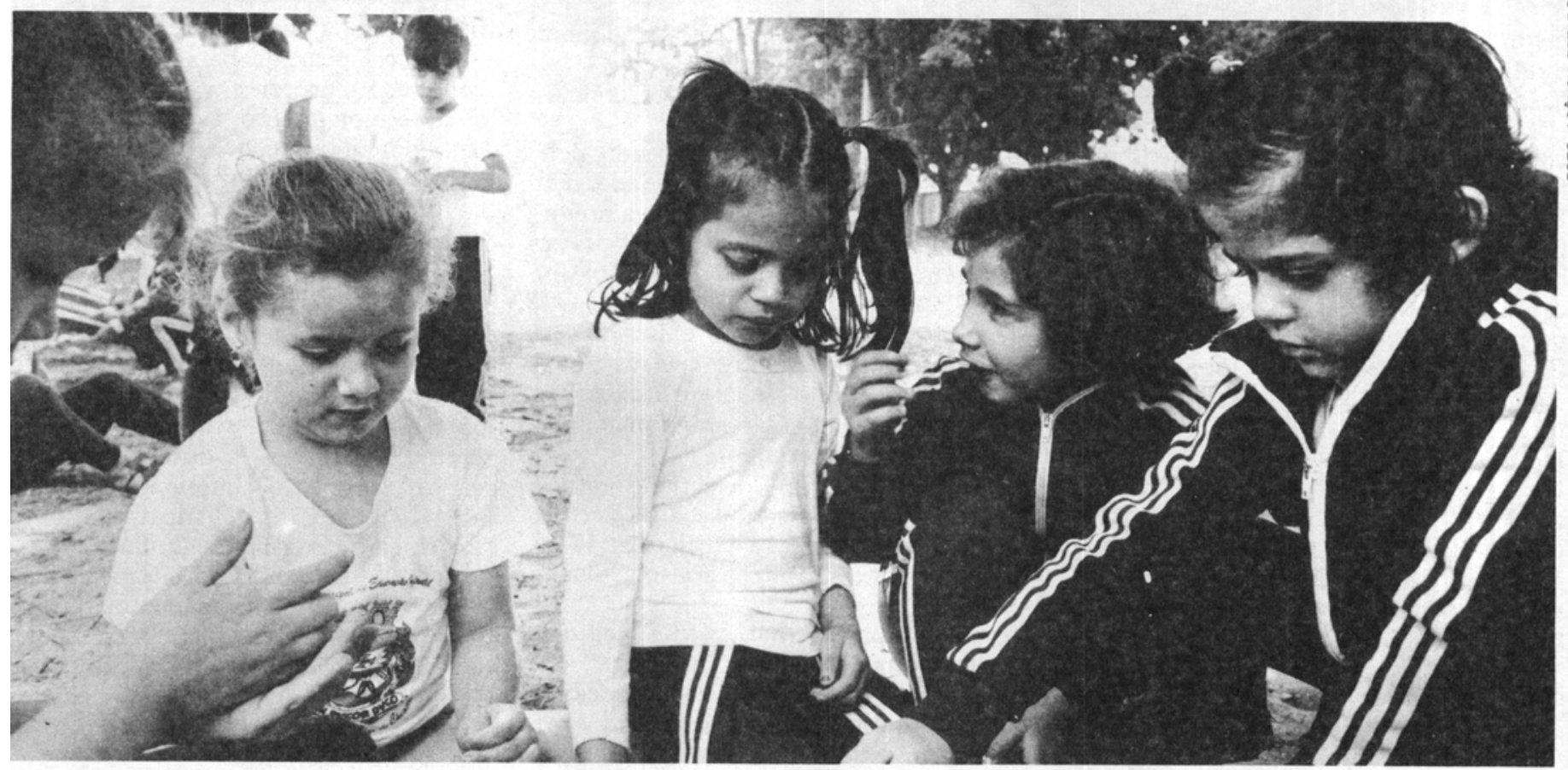

"O ambiente afeta o comportamento da criança.

Todos os programas procuraram ensinar à mãe, numa situaçâo prática, técnicas de interaçāo mãe-filho, alguns através de visitas domiciliares, em geral semanais (Ramey, Sparling, Wasik e Bryant, 1979; Honig 1977; Gray e Klaus, 1965), outros através de sessōes no próprio centro (Hayden, 1976; Fredricks, Baldwin e Grove, 1976; Wiegenrink e Parrish, 1976) ou ambos (Jones, 1977).

Com relação às differentes áreas de desenvolvimento abrangidas, os programas de Ramey, Sparling, Wasik e Bryant (1979), os de Garber (1977) e Gray e Klaus (1965) deram maior ênfase à linguagem, sendo que os dois últimos também incluíram aspectos cognitivos. Os programas de Hayden (1976) e Fredricks, Baldwin e Grove (1976), além dos aspectos cognitivos e de linguagem, enfatizaram também as áreas motoras $\mathrm{e}$ deauto-cuidado. Honig (1977) e Jones (1977) utilizaram um currículo Piagetiano.

Ramey, Sparling, Wasik e Bryant (1979), Garber (1977), Honing (1977) e Gray e Klaus (1965) utilizaram grupos experimentais e de controle, enquanto Hayden (1970), Fredricks, Baldwin e Grove (1976) e Wiegentink e Parrish (1976) utilizaram um desenho experimental de caso único.

Com relação à avaliação final, o programa de Ramey, Sparling, Wasik e Bryant avaliou o desenvolvimento da criança, o status intelectual dos irmāos, as atitudes dos pais com relação à educaçāo de crianças, a interaçāo mãe-filho através do "Home Observation for the Measu- rement of the Environment" e videotape de laboratório. Os demais programas utilizaram os seguintes instrumentos: Gesell e Piaget, Stanford-Binet, "Wechsler Preschool and Primary Scale of Intelligence". WISC, tarefas experimentais de aprendizagem, medidas de interaçāo mãe-filho, "Illinois Test of Psycholinguistic Abilities" (Garber, 1977); Cattell, Stanford-Bimet, Bayley, escalas de Piaget, "Early Language Assessment Scale" $\mathrm{e}$ "Cornell Descriptive Scanning Record of Infant Activity", até os 2 anos de idade (Honig, 1977); "Socialization Scale for Children", "Denver Development Screening Test", itens do Gesell e Vineland, assim como registros individuais dos objetivos e procedimentos (Jones, 1977).

Os principais resultados e conclusões desses trabalhos mostram que: - o sistema de interação mãe-fílho é modificável pela educagāo familiar, sen. do que a mudança parece estar na direção de um aumento na comunicaçāo e no envolvimento diádico (Ramey, Sparling, Wasik e Bryant, 1979; Garber, 1977).

- as diferenças no desenvolvimento dos grupos experimental e de controle começaram a surgir a partir dos $18 \mathrm{me}$ ses (Garber, 1977).

- os irmãos dos sujeitos do grupo experimental mostraram os mesmos resultados, ou seja, estagnação do declínio em seu desenvolvimento (Garber, 1977).

- os sujeitos do grupo experimental apresentaram um melhor desempenho (Garber, 1977; Honig, 1977; Jones, 1977).
3) Programas de intervenção realizados em casa, com envolvimento da mãe

Os programas aqui incluídos utilizaram apenas a mãe como agente de intervençāo de seus filhos. Neste caso, as crianças nāo freqüentaram nenhum centro ou creche no qual recebessem instruçāo por pessoal especializado.

O principal objetivo desses programas foi, de uma maneira geral, treinar a mãe a ser o principal agente de intervenção junto à criança, para prevenir um atraso em seu desenvolvimento (Montenegro et al., 1977; Adriasola et al., 1979; Badger, 1977; DEBT; Bromwich, 1981; Shearer e Shearer, 1972; Boyd, 1980 Cochran e Loftin, 1980; Levenstein, 1970).

Pode-se dizer que, na grande majoria desses programas, o principal critério de inclusão dos sujeitos foi o baixo status sócio-econômico da família. Entretanto, alguns indicaram diferentes critérios como déficit na fala e lingua. gem; problema físico, de audição, de visāo ou emocional; retardo mental e atraso no desenvolvimento e/ou qualquer outra condiçâo de alto risco (DEBT; Shearer e Shearer, 1972), prematuros com menos de 37 semanas e menos de $2,5 \mathrm{Kg}$, bebês deficientes, problemas de comportamento e com um leve atraso no desenvolvimento, e também sem nenhum problema observável mas com um baixo escore na avaliaçăo cumulativa de 0 a 9 meses de idade (Bromwich, 1981).

Os instrumentos para a avaliação inicial, antes de qualquer intervençāo, foram: observação informal em casa e dados de 
nistória de caso, parte do "Vineland Scale of Social Maturity", Denver, Koontz, Reel e avaliação médica (DEBT), 14 medidas fisiológicas e psicológicas entre os 0 e 9 meses de idade, "Parent Behavior Progression', "Play Interaction Measure" (interaçāo māefilho no laboratório), observaçöes do bebê e interaçāo mãe-filho em casa e no centro, informaçōes de conversas com os pais e da equipe médico-enfermeira (Bromwich, 1981), "Development Skill Age Inventory" de Alporn e Roll, Stanford-Binet (Form I-M) Cattell "Peabody Picture Vocabulary Test" e "Slossen Intelligence Test for Children and Adults" (Shearer e Shearer, 1972), "Parental Behavior Inventory" (Boyd, 1980), Cattell, Stanford-Binet e "Peabody Picture Vocabulary Test" (Levenstein, 1970; Madden, Levenstein e Levenstein, 1976).

Todos os programas utilizaram visitas domiciliares semanais ou quinzenais, para o treinamento da mãe, exceto o de Badyer (1977) que só utilizou reunióes de grupo no centro. Os programas de Badger (s.d.) e DEBT também utilizaram esse tipo de reunióes, além das visitas.

Com relaçâo ao procedimento de treino das māes, alguns programas utilizaram apenas a demonstração pelo visitador de como interagir com a criança (Montenegro et. al., 1977; Adriasola et al., 1977; DEBT) enquanto que outros além da demonstração utilizaram a modelagem do comportamento da mãe (Badger, 1977; Shearer, 1976). Os programas de Montenegro e Adriasola também envolveram a entrega, às màes, de 24 manuais mensais, contendo os exercícios que elas deveriam realizar com seus filhos.

Todos os programas incluíram as principais áreas de desenvolvimento, ou seja, cognitiva, motora, linguagem e social, sendo que o de Levenstein (1970) e Madden et al. (1976) deram ênfase especial à interaçắo verbal proporcionada pela mãe.

Os instrumentos de avaliação finnal foram os seguintes: questionário (Badger, s.d.); prova de Piaget, WAL.S, "Escala de Evaluación del Desarrolo Psicomotor" de Rodriguez, Arancibia e Undurraga (Montenegro et al., 1977; Adriasola et al., 1979); "Uzgiris-Hunt Infant Ordinal Scales of Psychological Development", Bayley (Badger, 1977); Denver e Koontz (DEBT); "Parent Behavior Progression", "Play Interaction Measure" e comportamento do bebê (Bromwich, 1981); registro dos pais, linha de base e pós-linha de base pelo visitador, Stanford-Binet, Cattell, Alpern e Boll, Gesell (Shearer e Shearer, 1972); "Parental Behavor Inventory" (Boyd 1980), Catell. Stanford Dinet e
Deabody (Levenstein, 1970) Maddenet al., 1976).

Os principais resultados e conclusões apresentados são:

- quando o currículo é aplicado pela primeira vez aos 15 meses de idade, nāo ocorre uma melhora significativa no desempenho da criança, a curto prazo (Montenegro et al., 1977; Adriasola et al., 1979).

- muitas vezes o grupo experimental adquire comportamentos, meses antes do grupo de controle, constituído por crianças de nível sócio-econômico médio (Montenegro et al., 1977).

- as diferenças no desenvolvimento dos grupos experimental e de controle começaram a surgir a partir dos 12-15 meses (Badger, 1977; Adriasola et al., 1979).

- muitos pajs tornaram-se funcionalmente independentes do visitador, em termos de planejamento da instruçăo e sua implementação (Boyd, 1980).

- os irmãos mais moços das crianças tratadas também foram beneficiados pelo programa, apesar de não terem sido submetidos a uma intervenção (Madden et al., 1976).

- houve uma proporção menor de crianças com problemas acadêmidos severos, em classes especiais, e uma proporção maior de crianças na classe adequada à sua idade (Levenstein, 1978).

- ocorre um melhor desempenho para os sujeitos do grupo experimental, naqueles programas que utilizaram esse tipo de desenho.

- constatou-se um progresso de $13 \mathrm{me}$ ses, em idade mental, num período de 8 meses, quando o progresso esperado seria de 6 meses (Shearer e Shearer, 1972).

\section{ANÁLISE $\mathrm{E}$ \\ IMPLICACุĀO DOS PROGRAMAS DE INTER VENÇÃo}

Alguns aspectos dos procedimentos dos diferentes programas de intervenção precoce precisam ser analisados para que uma melhor avaliação possa ser feita e para que novos rumos de investigação possam ser tomados.

Se forem aceitos como ponto de partida os objetivos implícitos, dos diferentes programas, notar-se-á que todos eles procuram ensinar comportamentos à criança. Mesmo que a mãe tenha sido envolvida no programa, o objetivo é fazer com que a mãe ensine à criança determinados comportamentos, em situaçōes arbitrárias. Parece que seria inicialmente essencial colocar o problema, para então fazer as perguntas necessárias e relevantes que poderão levar a uma solução apropriada. Seria possível afirmar que há uma maior probabilidade de que crianças de baixo nível sócioeconômico apresentem um atraso em seu desenvolvimento. É preciso perguntar-se por que isso ocorre ou quais sāo as diferenças nos ambientes das crianças de alto e baixo status sócioeconômico que podem ser responsáveis por esse atraso. Mas isto nāo tem sido feito.

Alguns estudos mostram a importância da variáveis da mãe sobre o desenvolvimento da criança, mas não mostram como isto está ocorrendo, em termos funcionais. A observação natural dos padrōes de interação mãe-filiho poderia, em parte, verificar como isso ocorre para determinada criança. Se for aceito que o padrão de interação mãefilho é um fator fundamental para o desenvolvimento da criança, entăo esse deve ser o principal foco dos programas de intervençāo, e não o ensino de determinados comportamentos ausentes do repertório da criança.

Esse tem sido o ponto de vista de Bromwich (1981) que, em seu programa, não utilizou nenhum currículo para planejar atjvidades para a criança, dando maior enfase as "prioridades, atitudes e habilidades dos pais" para a determinaçāo das atividades mais adequadas para a mãe e a criança.

As implicações de não proceder desta maneira, de fazer como a maioria dos programas, isto é, de elaborar um currículo de atividades, é que o comportamento que se quer ensinar à criança pode não ser funcional em seu ambiente, principalmente, naqueles programas onde a criança vai a uma creche diariamente, năo havendo portanto modificaçāo em seu ambiente natural. Mas também nos programas que procuram envolver a mãe é preciso perguntar-se o que está ocorrendo nas interaçöes māefilho, entre as sessões de treino ou ensino. Em outras palavras, como a mäe se comporta quando ela nāo está específicamente envolvida na tarefa determinada pelo curtículo? A estimulaçăo da criança pode e deve ser levada a cabo em qualquer rotina e não apenas durante determinado período do dia. Isto é fundamental para que ocorram a generalização e manutenção dos comportamentos, não só da criança como também da mãe. Contudo esses fatores não têm sido levados em consideração, na maior parte dos programas, o que provavelmente resultou na freqüente diminuição dos efeitos, tal como relatado em avaliaçôes da literatura (Horowitz, 1980; Ramey e Gowen, 1980; Bronfenbrenner, 1976). Para Ramey e Gowen, efeitos duradouros podem ser obtidos se o ambiente de casa for modificado, sen- 
do a forma de interação mãe-filho o fator mais consistentemente relacionado ao desempenho subseqüente da criança. Esses dois autores também consideram haver uma associaçăo entre desempenho da criança e quantidade de estimulaçāo da linguagem, assim como práticas positivas e responsivas de interação com a criança.

Apesar disso, alguma generalizaçāo dos efejtos dos procedimentos te m ocorrido, já que alguns programas que utilizam a mäe como agente de intervenção têm demonstrado que os irmāos mais moços do sujeito do programa também passam a apresentar um desenvolvimento dentro dos padrōes "normais" e já que os efeitos desses programas são mais duradouros, se comparados aos que năo incluem o envolvimento da mãe.

E preciso considerar, ainda, que năo só o comportamento da criança é funçāo do comportamento da mãe, mas que também o comportamento desta é função do comportamento da criança. Zeskind e Ramey (1978) afirmam que bebês pouco responsivos podem ter mães menos responsivas e sensíveis às suas açōes. Isto está de acordo com os resultados de um estudo realizado no México e mencionado por Brozek (1979), no qual se verificou que as crianças desnutridas que tiveram sua dieta suplementada eram mais ativas, tinham um repertório comportamental mais amplo, provocando uma interação mais intensa com seus pais. $O$ resultado desses dois estudos também vem mostrar a influência do comportamento da própria criança sobre o comportamento daqueles que cuidam dela e, portanto, a importância de uma análise funcional da situação de interaçāo que não pode ser ignorada ao elaboraj-se um programa de intervenção.

Com relação ao comportamento da criança em desvantagem e o da privilegiada, não se acredita que o comportamento de uma possa ser considerado superior ou inferior ao da outra. Gray e Klaus (1965) e Ramey, Sparling, Wasik e Bryant (1979) consideram que essa diferença é mais qualitativa do que quantitativa, sendo possivel que a mãe da criança em desvantagem esteja comportando se de maneira tão apropriada e sensivel com seus filhos quanto a da criança privilegiada que se comporta de maneira diferente. Daí a importância dos programas individualizados. Em sua maioria os programas atuais podem ser considerados individualizados no sentido de ensinar à criança aqueles comportamentos que ela ainda não possui, mas năo săo individualizados no sentido de procurar avaliar por que aquela criança não apresenta aquele ou aqueles comportamentos em seu repertório, isto é, sua inserção no contexto funcional de padrões de interação mãe-filho.

Em síntese, vê-se que uma deficiência na abordagem inicial (a criança em desvantagem tem um repertório comportamental restrito, em vez de a criança em desvantagem ter um ambiente diferente) leva à adoção de um procedimento deficiente (procurar estabelecer no repertório comportamental dessa criança aqueles comportamentos ausentes e que são apresentados pela maioria das crianças privilegiadas, em vez de procurar tornar seu ambiente tão favorecedor ao desenvolvimento quanto o da criança privilegiada). Em decorrêncja disso, encontram-se, na maioria dos programas, dois outros problemas metodológicos, que serāo analisados a seguir. Trata-se da utilizaçăo de testes de QI, para a avaliaçāo, e do desenho experimental de grupos.

A utilização de testes de QI, como método de avaliação da capacidade de uma criança, tem sido criticada por diversos autores (Ashton-Lilo, 1979; Bromwich, 1981; Greenspan, Nover, Silver e Lourie, 1979; Bronfenbrenner, 1976). Primeiro, pela própria situação de aplicação de testes, que é inteiramente artificial. Nestas condiçōes, e principalmente quando se tratam de crianças muitas pequenas, é muito dificil conseguir a colaboração do sujeito, devido a motivos tais como cansaço, irritaçào, doença e estranheza do aplicador. Além disso, se a criança nào realiza a tarefa em determinado lugar, com determinada pessoa e em determinado momento, isto nāo significa que ela não é capaz de fazê-lo. Zigler, Abelson Trickett e Seitz (1980) dizem que os escores convencionais de QI podem ser indicadores menos válidos do funcionamento cognitivo das crianças em desvantagem por causa de diferenças culturais e porque todo pósteste apresenta sempre um aumento de 10 pontos de QI, mesmo que o programa só tenha durado umas 8 semanas. Eles afirmam que há uma evidência crescente de que o desempenho das crianças em desvantagem é afetado desfavoravelmente por fatores motivacionais e enfatizam a importância de manter a distinçăo entre capacidade e desempenho. Neste caso, o escore de QI seria uma base questionável para julgar o valor dos programas de intervenção.

Além do mais, embora seja impor. tante compreender o desenvolvimento e suas seqüências, determinar somente 0 que a criança pode fazer e quais as habilidades que devem vị em seguida parece ser insuficiente. A questảo năo é saber o que a criança pode ou não pode fazer, como medido pelos testes, mas saber como o ambiente de determinada criança está afetando seu comportamento e como ela está respondendo a esse ambiente. A utilizaçāo exclusiva de um teste não dá uma visão da habilidade funcional da criança com seu ambiente ou da familia para lidar com a criança. Se utilizarmos um teste e, além disso, a observação natural, verificar-se-á que a criança é capaz de fazer muitas outras coisas que não são avaliadas pelo teste. Soares da Silva (1979) mostrou que, depois da intervenção, 2 sujeitos apresentaram uma grande methora em seu comportamento exploratório-manipulativo, medido através de um registro contínuo específico e de um registro gravado, enquanto os dados obtidos através do Koontz não mostraram essa melhora.

Finalmente, serão analisadas as ljmitaçōes da utilização de um desenho experimental de grupos. $O$ que os dados desse tipo de desenho podem dizer é que a média dos resultados do grupo experimentál foi significativa em contraposição à média dos resultados do grupo de controle. Mas o resultado médio de um grupo pode ou não representar o desempenho de um indivíduo do grupo. Além disso, pode ocorrer que um tratamento que é estatisticamente significativo não seja clinicamente significativo, ou seja, o tratamento é eficaz, mas não o bastante. A significância estatística também pode subestimar a eficácia do tratamento para algum sujeito experimental (Hersen e Barlow, 1977). Finalmente, perde-se sempre em termos de generalização dos resultados, para outros indivíduos, tanto no caso do grupo ser mujto homogêneo ou muito heterogêneo, isto é, há uma dificuldade de generalizar os resultados assim obtidos para sujeitos com outras características. assim como para sujeitos com características muito especificas, respectivamente.

Em síntese, quando se aplica determinado procedimento para determinada criança, o que interessa é saber se esse procedimento está sendo eficaz para essa criança e fazer as modificações apropriadas, quando necessário. Existe uma quantidade enorme de variáveis infłuenciando o comportamento, de maneira que seria ingênuo supor que um programa-padräo fosse eficaz para todos os sujeitos. Seria difícil imaginar um caso em que não fosse necessário recorrer a uma análise individual dos dados. Uma vantagem dos desenhos de caso único é que o investigador pode comecar a procura imediata para a causa da tendência comportamental, alterando ao mesmo tempo seu desenho experimental. 


\section{REFERÊNCIAS BIBLIOGRÁFICAS}

Adriasola, A., Arancibia, V., Marín, P. e Adriasola; G. Progresando la Calidad de le Vida Hurmana. Estimulación Psicosocial Temprama.

Universidad de Chile, Facultad de Medicina, 1979.

Ashton-Lilo, L.J. Infonts and their

Environment: Working with High Risk Infants and Parents. Paper presented at the sth International Congress of the International Association of Scientific Study of Mental Deficiency, Jerusalem, lsrael, 1979.

Badger, E. The Infant Stimulation/Mother Training Project. In Caldewell, B.M. e Stedman, D.J. (org.), Infent Education: A Guide for Helping Handicapped Children in the First Three Years. New York, N.Y.: Walker and Company, 1977.

Badger, E. Mothers Training Program:

Educational Intervention by the Mothers of Disadvantaged Ininnts.

Boyd, R.D. Systematic Parente Training through a Home-based Model. Portinge Project Readings. Portage, Wis.: The Portage Project, 1980.

Bromwich, R. Working with Parents and Infents, An Interactional Approach. Baltimore: University Park Press, 1981.

Brcif:nbrenner, U. Is Early Intervention Effective? A Report on Longitudinal

Evaluations of Preschood Programs, vol. 2. Department of Health, Education, and Welfare. DHEW Publication $n^{\circ}(\mathrm{OHD})$ 76-30025.

Brozek. J.Nutriçāo. Desnutriçāo e

Comportamento. Separata de Cadernos de Pesquisa. Fundaçāo Carlos Chagas, 29 jun. 1979. Cocharan, D. e Loftin, C. The Portage Model in the Head Start Homebased Option, Portage Project Readings. Portage, Wis.: The Portage Project, 1980.

DEBT Gospel. Developmentaf Education Birth through Two. Lubbock Independent School Distric, Lubbock, Texas.

Fredricks, H.D., Baldwin, V.L. e Grove, D. A Home-center Based Parent Training Model. In Lillie, D.L. Trohanis, P.L. (org.), Teachlna

Parents to Teach, New York, N.Y.: Walker and Company, 1976

Garber, H.L. Preventing Mental Retardation through Family Rahabilitation. In Caldwell,

B.M. e Stedman, D.J. (org.), Infant Education: A Guide for Helping Handicapped Children in the First Three Years. New York, N.Y.: Walket and Company, 1977.

Gray, S.W. e Klaus, R.A. An Experimental Preschool Program for Culturally Deprived Children. Child Development, 1965, 36, 887-898. Greenspan, S.I., Nover, R.A., Silver, B.J. e Lourie, R.S. Methodology Issues and an Approach to Assessment in Clinical Infants Programs. In Clinical Infant Intervention Research Programs. Selected Overview and Discussion. Department of Health, Education, and Welfare. DHEW Publication $n^{\circ}$ (ADM) 79-748.

Hayden, A.H. A Center-based Parent-training Model. In Lillie, D.L. e Trohanis, P.L. (org.)

Teaching Parents to Teach. New York, N.Y.:

Walker and Company, 1976.

Hersen, M. e Barlow, D.H. Single-case

Experimental Desians: Strateojes tor Studying Behavior Change. Oxford: Pergamon Press, 1977.

Honig, A.S. The Children's Center and the Family Development Research Program. In Caldwell, B.M. e Stedmen, D.J. (org.), Imfant Education: A Guble for Helping Handicapped
Children to the First Three Years. New York,

N.Y.: Walker and Company, 1977.

Horowitz, F.D. Intervention and its Effects on

Early Development: What Model of

Development is Appropriate? In Liferpen

Developmentel Peychology. Intervention. New

York, N.Y.: Academic Press, 1980.

Jones, M.H. Intervention Programs for

Children under Three Years, In Caldwell, B.M

e Stedman, D.J. (org.), Inlant Education: A

Gulde for Helping Handicapped Children in the

First Three Years. New York, N.Y.: Walker

and Company, 1977.

Levenstein, P. Cognitive Growth in

Preschoolers through Verball Interaction with

Mothers. American Journal of Orthopsychintry,

$1970,40,426-432$.

Levenstein, P. Third Grade Effects of the

Motber-Child Home Program. Final Report,

manuscrito náo publicado, 1978.

Madden, J., Levenstein, P. e Levenstein, S.

Longituidinal IQ Outcomes of the

Mother-Child Home Program. Child

Development 1976, 47, 1015-1025.

Montenegro A., H., Rodríguez S., S., Lira L.,

M.I., Haeussler P., I.M. e Braliō E., S.

Programa Piloto de Estlmulación Precoz para

Ninö́s de Nivel Socloeconómico Bajo entre 0 y 2

año. Informe final. Servicio Nacional de

Salud, Sección Salud Mental, Santiago, Chile,

1977.

Ramey, C.T. e Campbell, F.A. Compensatory

Education for Disadvantaged Children, School

Review, 1979, 87 171-189. (a)

Ramey, C.T. e Campbell, F.A. Early

Childhood Education for Psychosocially

Disadvantaged Children: Effects on

Psychological Processes. American Journal of

Mental Deficlency, 1979, 83, 645-648. (b)

Ramey, C.T., Farran, D.C. e Campbell, F.A

Predicting IQ from Mother-Infants

Interactions. Child Development, 1979, 50 ,

804-814.

Ramey, C.T. e Gowen, J.W. Children at Risk:

Identification and Intervention. Children

Today, 1980, 9, 12-16.

Ramey, C.T., Sparlin, J.J., Wasik, B.H. e Bryant, D. A Model Fort Educenting parents of high-risk Infants. Paper presented at the Ira J. Gordon Memorial Conference on Parent Education and Involvement. Chapel Hill, N, Carolina, 1979.

Shearer, M.S. A home-based parent-training Model. In Lillie, D.L. e Trohanis, P.L. (org.), Teaching parents to Teach.

New York, N.Y.: Walker and Company, 1976. Shearer, M.S. C Shearer, D.E. The Portage Projetct: A Model for Early Childhood Education. Exceptional Children, 1972, 36, 210-217.

Soares da Silva, V.L. Desenvolvimento de um Programa de Treinamento do Comportamento Exploratório-Manipulativo em Crianças, strovés de Estimulaçấ Ambiental e Reforçmento Social. Dissertaçâo de Mestrado, Universidade de Basília, 1979.

Wiegenrink, R. e Parrish, V. A Parent Implemented. Preschool Program. In Lillie, D.L. e Trohanis, P.L. (org.), Teaching Parents to Teach. New York, N.Y.: Walker and Company, 1976. Zeskind, P.S. e Ramey, C.T. Fetal Malnutrition: An Experimental Study of its Consequences on Infant Development in Two Caregiving Environments. Child Development, $1978,49,1155-1162$.

Zigler, E., Abelson, W.D. Trickett, P.K. e Seitz, V. Is an Intervention Program Necessary In Order to Improve Economically-disedvantaged Children's iQ Scores? Manuscrito nāo publicado. Yale University, New Haven, Conn, 1980. do livro de Regina Vieira

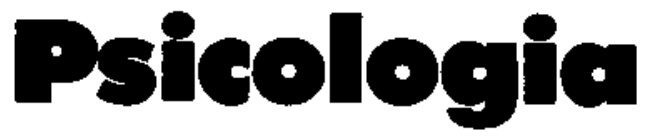

Célia Maria Lana da Costa Zannon

Universidade de Brasilia

Escrito para mães, em uma linguagem simples e de fácil compreensáo para o leitor leigo, este livro é proposto pela autora como um auxilio para a reflexão sobre dúvidas e soluçōes encontradas pelos pais no relacionamento com seus filhos nos dois primeiros anos de vida.

A autora salienta, dentre seus objetivos, a divulgação da importância prática da Psicologia do Desenvolvimento, caracterizando seu livro como uma fonte de informação para a compreensão do comportamento da criança.

Baseia-se em proposiçōes de autores como Fenichel, Gesell, Piaget, Rogers, Spitz e Winnicott, em manuais tradicionais de Desenvolvimento da Criança (Hurlock, Jersild, Mira Y Lopes, Mussen Conger e Kagan), em opiniões de pediatras como Delamare, Salk e Spock e em suas próprias experiếncias como Psicóloga na área clínica infantil.

A organização dos capítulos confere ao texto uma estrutura que, embora nāo explicitada pela autora, adquire relevância, visto que encerra uma orientação e uma hierarquização da importância dos diferentes agentes sociais responsáveis pelos cuidados da criança nos primeiros anos de vida.

Poder-se-ia dizer que o livro tem três partes. Os dois capitulos iniciais, sobre a relaçảo mãe-criança e o papel do pai, compỏem uma parte dedicada aos primeiros e principais agentes adultos no desenvolvimento da criança pequena. Os dois capitulos seguintes comporiam uma segunda parte, de descriçāo das mudanças comportamentais no primeiro e no segundo anos e de apresentação de problemas e dúvidas mais comuns nos diferentes estágios do desenvolvimento. No que seria a parte final, a autora retoma a questāo dos agentes sociais e apresenta, em dois capítulos, aspectos referentes aos cuidados por parentes (tios e avós) e por babás, no contexto da necessidade de separaçāo mâecriança.

$O$ texto tem aspectos positivos e relevantes em uma proposta de orientaçāo: o destaque dos problemas comumente rela. tados pelos pais no contexto do atendjmento clínico; a atençăo à variedade de situaçōes e variáveis que podem estar envolvidas em vários dos problemas relatados; a apresentação de alternativas diferentes de soluçâo para alguns problemas; a busca de suporte no conhecimento acumulado na área de Psicologia de Desenvol. 